\title{
The efficacy of combination therapies including antiviral drugs, methylprednisolone and daily proning in 20 patients with COVID-19 requiring invasive mechanical ventilation-Case series in a single critical care center in Osaka, Japan
}

Haruka Shimazu ( $\sim$ kukki.lucky.candy@gmail.com )

Kansai Medical University Medical Center

Kazuhisa Yoshiya

Kansai Medical University Medical Center

Keisuke Tamagaki

Kansai Medical University Medical Center

Yasutaka Okamoto

Kansai Medical University Medical Center

Hitoshi Nakano

Kansai Medical University Medical Center

Hiroto Okubata

Kansai Medical University Medical Center

Yumiko Miyano

Kansai Medical University Medical Center

Shuhei Matsunami

Kansai Medical University Medical Center

Shuhei Maruyama

Kansai Medical University Medical Center

Shuji Kanayama

Kansai Medical University Medical Center

Hiromu Iwamura

Kansai Medical University Medical Center

Daiki Wada

Kansai Medical University Medical Center

Tomoyuki Yoshihara

Kansai Medical University Medical Center

Fukuki Saito

Kansai Medical University Medical Center 


\section{Yasushi Nakamori}

Kansai Medical University Medical Center

\section{Yasuyuki Kuwagata}

Kansai Medical University Hospital

\section{Research}

Keywords: COVID-19, favipiravir, Methylprednisolone, Mycosis, Proning, invasive mechanical ventilation

Posted Date: July 16th, 2020

DOI: https://doi.org/10.21203/rs.3.rs-41984/v1

License: (c) (1) This work is licensed under a Creative Commons Attribution 4.0 International License. Read Full License

Version of Record: A version of this preprint was published at Archives of Clinical and Medical Case Reports on January 1st, 2020. See the published version at https://doi.org/10.26502/acmcr.96550274. 


\section{Abstract}

Background: Since December 2019, the COVID-19 infection has drastically spread across China and the world, including Japan. Few reports so far have clarified the prognosis and treatment of critically ill patients managed with invasive mechanical ventilation. This study aimed to present the clinical courses of 20 critically ill patients with invasive mechanical ventilation, which may be valuable for determining future therapies and intensive care of critically ill patients with COVID-19.

Methods: In this observational, single-center, cohort study, we included 20 critically ill patients with laboratory-confirmed SARS-CoV-2 infection who were admitted to our hospital ICU and required invasive mechanical ventilation. The patients' general characteristics, laboratory data, treatments, and outcomes were assessed between survivors and non-survivors.

Results: Among these 20 patients, 14 patients survived and 6 patients died. The lowest lymphocyte count (93 vs $279 / \mu \mathrm{L}, \mathrm{p}<0.01$ ) and the lowest platelet count (12 vs $152 \times 10^{3} / \mu \mathrm{L}, \mathrm{p}<0.01$ ) were significantly lower, and the highest KL-6 value (1584 vs $546 \mathrm{U} / \mathrm{mL}, \mathrm{p}=0.02$ ) was significantly higher, in the non-survivor group versus the survivor group during the patients' ICU stay. In addition to antiviral treatments and daily proning of the patients, methylprednisolone was administered to all patients to control cytokine storm syndrome following the virus infection. Six patients died from complications such as fungal infection, but no patients died of respiratory failure. As a result, none of the patients required ECMO.

Conclusion: This report described the prognosis of COVID-19 patients required invasive mechanical ventilation in a single Japanese critical care center. Multidisciplinary treatments using a single protocol, including antiviral therapies, anti-inflammatory therapies, and respiratory physiotherapies, were effective for critically ill COVID-19 patients who required invasive mechanical ventilation.

\section{Introduction}

In December 2019, an outbreak of severe acute respiratory syndrome coronavirus 2 (SARS-CoV-2) infection began in Wuhan city, the capital of Hubei province in China. Since then, the virus has drastically spread across China and the world, and this infection was later designated coronavirus disease 2019 (COVID-19) by the World Health Organization (WHO) [1]. As of May 31, 2020, a total of 5,939,234 cases of SARS-CoV-2 infection have been confirmed, and 367,255 people have died around the world according to WHO [2]. In Japan, the Ministry of Health, Labor and Welfare reported that 16,884 cases of SARS-CoV-2 infection had been detected by May 31, 2020, and 892 patients had died [3].

SARS-CoV-2 infection starts with an interaction with angiotensin converting enzyme 2 (ACE2). The virus binds to and invades cells that express ACE2, such as type II alveolar cells of lung, kidney proximal tubule cells, myocardial cells and enterocytes of the ileum and colon, and causes various organ dysfunctions including lung injury and acute respiratory distress syndrome (ARDS) [4]. Infection of SARS-CoV-2 is thought to secondarily cause macrophage activation syndrome and hemophagocytic lymphohistiocytosis, which are characterized by cytokine storm syndrome accompanied by multiple 
organ failure [5]. Thus far, there are no specific therapeutic agents for COVID-19. Therefore, to treat severely ill patients with COVID-19, in addition to antiviral therapies, control of hypercytokinemia due to macrophage activation syndrome and hemophagocytic lymphohistiocytosis is also important.

The clinical courses of COVID-19 range from asymptomatic to critically ill, and most patients appear to have a favorable prognosis. However, the prognosis of critically ill patients, especially mechanically ventilated patients, is unclear. Studies from China [6, 7], Italy [8], the US [9] and Canada [10] showed mortality rates ranging from $15-62 \%$ in critically ill patients. In these studies, many patients were still in the ICUs, and the exact mortality may actually be worse than has been reported. There are no reports, to our knowledge, on the prognosis of patients with invasive mechanical ventilation. It is important not only to prevent the patients' condition from worsening but also to determine how to treat and save critically ill patients who require invasive mechanical ventilation.

In this study, we present the clinical courses of 20 critically ill patients with COVID-19 treated with invasive mechanical ventilation. We believe that the data from this study may be valuable for determining future therapies and intensive care of critically ill patients with COVID-19.

\section{Methods}

\section{Study design and participants}

This was an observational cohort study conducted at a single urban, academic, critical care center in Osaka, Japan, between February 1 and May 31,2020. We included severely ill patients with laboratoryconfirmed SARS-CoV-2 infection who were admitted to our hospital ICU and required invasive mechanical ventilation. We excluded patients with DNR code status and cardiopulmonary arrest on admission. SARSCoV-2 infection was defined by a positive result of reverse transcriptase polymerase chain reaction (RTPCR) detection of a specimen collected on a nasal/oropharyngeal swab or sputum. This study was approved by the ethics committee of Kansai Medical University General Medical Center and followed the principles of the Declaration of Helsinki. No informed consent was required, and researchers analyzed only deidentified data.

\section{Procedures}

We conducted the treatments according to our intensive care COVID-19 management algorithm as follows: 1) Invasive mechanical ventilation (pressure control ventilation mode with target tidal volume of 6-8 $\mathrm{mL} \cdot \mathrm{kg}^{-1}$, positive end-expiratory pressure of $5-20 \mathrm{cmH}_{2} \mathrm{O}$, and plateau pressure of less than 30 $\mathrm{cmH}_{2} \mathrm{O}$ ); 2) therapeutic agents for COVID-19 (favipiravir, ciclesonide, nafamostat mesilate, and methylprednisolone); 3) prophylactic antibacterial drug; 4) antifungal drug if necessary; 5) catecholamine support if necessary; 6 ) hemodialysis if necessary; 7) prone position as possible; and 8) extracorporeal membrane oxygenation (ECMO) when the patients' ratio of the partial pressure of arterial oxygen to the fraction of inspired oxygen (P/F ratio) is continuously below 100. 
Twenty patients who required invasive mechanical ventilation were identified (Table 1). The patients were divided into 2 groups (survivors group and non-survivors group), and a comparative review of the two groups was made statistically as described below. General data, including patient characteristics, were recorded retrospectively from the patients' electronic medical records. Coexisting disorders were obtained from physician's documentation, and the Acute Physiology and Chronic Health Evaluation II (APACHE II) score and Sequential Organ Failure Assessment (SOFA) score were calculated based on the disorders recorded.

Patients were assessed on admission and during ICU hospitalization. Laboratory data and the P/F ratio were recorded. Laboratory data included complete blood counts and values of plasma D-dimer, serum ferritin, sialylated carbohydrate antigen Krebs von den Lungen (KL-6), hepatic transaminase, creatine kinase, lactate dehydrogenase, and lactate. Treatment outcomes were length of the ICU stay, duration of invasive mechanical ventilation, and complications during hospitalization.

All radiological assessments and findings, including chest radiography and computed tomography (CT), were performed at the discretion of the treating physicians.

\section{Statistical analysis}

Descriptive statistics were used to summarize the data. Continuous variables are reported as the median and interquartile range (IQR), and categorical variables are summarized as counts and percentages. The Mann-Whitney $U$ test and Fisher's exact test were used, as appropriate, to compare differences between the 2 groups. A p-value of $<0.05$ was considered to indicate statistical significance. Statistical analyses were performed with SPSS for Windows version 22.0 software (SPSS, Inc., Chicago, IL, USA).

\section{Role of the funding source}

The funder had no role in study design, data collection, data analysis, data interpretation, or writing of this report. The corresponding author had full access to all data in the study and had final responsibility for the decision to submit for publication.

\section{Results}

\section{Patient characteristics}

From March 24 to May 10, 2020, 42 patients with moderate or severe COVID-19 were transported to our ICU, of whom 21 required invasive mechanical ventilation. Seven patients were transferred to our hospital after being intubated at another hospital. Fourteen patients with suspected or confirmed COVID-19 were admitted to our hospital and intubated in our ICU because of worsening oxygenation and severe findings on chest CT. One patient was excluded from this study because he tested positive for SARS-CoV-2 IgG antibodies but negative for SARS-CoV-2 by RT-PCR test. Thus, 20 patients were included in this study who required invasive mechanical ventilation and had laboratory-confirmed COVID-19. All patients were observed until they recovered and left the ICU, were transferred to another hospital without the need for 
mechanical ventilation, or died. Fourteen patients were alive and recovered from COVID-19 (survivor group), and 6 patients died (non-survivor group) in our hospital. The characteristics of the 20 patients are shown in Table 1. There is no significant difference in each variable except age is younger in survivor group than in non-survivor group.

\section{Laboratory examinations}

We compared the laboratory data of the 14 survivors and 6 non-survivors (Table 2 ). There was no significant difference between the two groups in the laboratory data on admission, including APACHE II and SOFA scores. However, the lowest lymphocyte count (93 vs 279/ $\mu \mathrm{L}, \mathrm{p}<0.01$, Mann-Whitney U test) and the lowest platelet count (12 vs $152 \times 10^{3} / \mu \mathrm{L}, \mathrm{p}<0.01$ ) were significantly lower, and the highest $\beta$-D glucan value (368 vs $4.5 \mathrm{pg} / \mathrm{mL}, \mathrm{p}<0.01)$ and the highest $\mathrm{KL}-6$ value $(1584 \mathrm{vs} 546 \mathrm{U} / \mathrm{mL}, \mathrm{p}=0.02)$ were significantly higher, in the non-survivors versus survivors during their ICU stay.

\section{Antiviral treatments}

We performed treatments for COVID-19 following our own algorithms. Favipiravir, ciclesonide, azithromycin, and nafamostat mesilate, which are expected to have antiviral effects, were administered to all patients. One patient received lopinavir/ritonavir only on the first day because we could not prepare favipiravir, but from the second day, we started favipiravir instead of lopinavir/ritonavir.

Hydroxychloroquine sulfate was administered to one patient before transfer to our hospital.

\section{Anti-inflammatory treatments}

We used methylprednisolone to control cytokine storm syndrome following the virus infection. With reference to chest radiographic findings and the severity of hypoxemia, methylprednisolone was started at $125 \mathrm{mg}$ per day or $1000 \mathrm{mg}$ per day. In the non-survivors, the administration period of methylprednisolone was prolonged and the cumulative doses were increased, as shown in Table 3 . We also regard tocilizumab as a candidate drug to control cytokine storm syndrome, but we could not start using it in our hospital until later in the study period. Accordingly, tocilizumab was administered to 3 patients before intubation, and 2 of them received tocilizumab before transfer to our hospital.

\section{Respiratory therapies}

We treated the 20 critically ill patients with therapeutic agents and invasive mechanical ventilation with daily prone positioning according to our own algorithm. Six of the 20 patients died from complications, but no patients died of respiratory failure. Our algorithm includes ECMO when the patient's P/F ratio is continuously below 100 , but daily proning and methylprednisolone therapy increased the P/F ratio above 100 and accordingly, we did not use ECMO in any of the patients.

The transition of mechanical ventilation parameters (lowest P/F ratio, plateau pressure, and positive endexpiratory pressure) from day 1 to 10 is shown in Fig. 1. There were no significant differences in each parameter between the survivor group and non-survivor group. 
Nineteen patients were placed in a complete prone position for 16 consecutive hours every day until their oxygenation improved. As Table 3 shows, the intubation period of the survivors was 10 (IQR: 7-12) days, duration of stay in ICU was 14 (IQR: 10-16) days, and proning was performed for 7 (IQR: 5-9) days. Each period above was significantly shorter than that of the non-survivors $(p<0.01)$.

No patients received neuromuscular blockade except at the time of intubation. Tracheostomy was performed in only one survivor.

\section{Antibiotic treatments}

Antibiotics were given prophylactically in all cases. To prevent nosocomial infection of the virus, bacterial culture tests other than blood culture were avoided in our hospital. Therefore, broad-spectrum antimicrobial (e.g., tazobactam/piperacillin) and anti-MRSA (e.g., linezolid) drugs were used. The incidence of fungal infection, which is considered to be a catheter-related bloodstream infection, was significantly higher in the non-survivors. Antifungal drugs were administered to 4 of the 6 non-survivors diagnosed as having mycosis by blood culture or increasing serum levels of $\beta-D$ glucan.

( $66 \%$ vs $7 \%, p<0.01$, Fisher's exact test)

\section{Complications}

Complications are listed in Table 3. Acute renal failure requiring hemodialysis $(100 \%$ vs $14 \%, p<0.01$, Fisher's exact test) and mycosis ( $66 \%$ vs $7 \%, p=0.01)$ occurred significantly more frequently in the nonsurvivors. Mycosis affected the prognosis of the non-survivors.

\section{Representative case}

The treatment course of a representative patient is illustrated in Fig. 2.

\section{Discussion}

This single-center case series describes 20 consecutive patients admitted to ICU during the first wave of the SARS-CoV-2 infection in Japan who required invasive mechanical ventilation. Our hospital is located in Osaka, Japan, with a population of 8.8 million. In Osaka, we expected to have 300 intubated patients during the peak period, and our hospital had prepared 20 beds for severe COVID-19 in the ICU. In actuality, the maximum number of intubated patients treated at the same time was 10 , and we were able to provide sufficient intensive care in all cases without the collapse of medical services. A treatment protocol was prepared for critically ill patients after referring to the papers based on experiences with Wuhan patients and those from the cruise ship Diamond Princess. We used favipiravir, nafamostat mesilate, ciclesonide, and azithromycin in combination as antiviral drugs. If oxygenation could not be maintained by usual invasive mechanical ventilation and antiviral therapy, we administered methylprednisolone according to ARDS treatment and daily proning therapy. If oxygenation worsened despite methylprednisolone and proning, we would initiate ECMO. 
The mortality rate of patients with COVID-19 who require invasive mechanical ventilation is not clear. In an early report from Wuhan, all 25 patients who required invasive mechanical ventilation died [11]. The mortality rates of patients requiring treatment in an ICU were reported from Washington State in the US [9], Wuhan in China [7], and the Lombardy region of Italy [8];, however, these reports did not mention the exact mortality rate of patients received invasive mechanical ventilation. A large multicenter study reported on 5700 patients requiring hospitalization for COVID-19 in New York [12]. Among 2634 patients who were discharged or died at the study endpoint, $88.1 \%$ of 320 patients who received invasive mechanical ventilation died. However, 831 intubated patients remained in hospital, and the prognosis of the intubated patients cannot be determined. In our small series, the mortality rate of the patients receiving invasive mechanical ventilation was $30 \%$. The reason for the lower mortality rate than those in the past reports is that medical services did not collapse in Japan, and the combination of antiviral, methylprednisolone, and proning therapies was effective. Especially, methylprednisolone and proning therapies effectively improved the patients' respiratory condition.

Our most anticipated antiviral drug is favipiravir, which is an RNA polymerase inhibitor developed as an anti-influenza drug and has been reported to have growth inhibitory activity against SARS-CoV-2 [13]. In a non-randomized study conducted in China, patients receiving favipiravir and interferon a had a faster negative PCR result for SARS-CoV-2 and faster improvement of CT findings than those receiving lopinavir/ritonavir and interferon a [14]. We administered favipiravir from the first day in all patients at a dose of $1800 \mathrm{mg}$ on the first day and $800 \mathrm{mg}$ on the second and subsequent days. We used favipiravir for 2 weeks or until the patients received a negative PCR result for SARS-CoV-2 or serious side effects occurred. In Japan, an observational study on COVID-19 patients treated with favipiravir was started in February 2020, and the results are anticipated.

Nafamostat mesylate is one of the serine protease inhibitors and has been used in Japan as an anticoagulant during hemodialysis and as an agent for disseminated intravascular coagulation. It inhibits the process of membrane fusion of SARS-CoV-2 and cells in vitro and is expected to have an antiviral effect on SARS-CoV-2 [5]. In addition, COVID-19 is reported to be associated with thrombosis, and prophylactic administration of anticoagulants is recommended in all hospitalized patients [15]. In anticipation of its antiviral and anticoagulant effects, we administered nafamostat mesylate for 1 week in all patients.

Ciclesonide is a safe drug widely used as an inhaled steroid for bronchial asthma and is expected to have local anti-inflammatory and viral replication inhibitory effects on COVID-19 [16]. We administered ciclesonide $800 \mu \mathrm{g}$ daily from a reservoir connected to the ventilator circuit for 2 weeks in all patients.

Some patients struck severely by SARS-CoV-2 might suffer cytokine storm syndrome that can lead to multi-organ failure including ARDS [5]. Conquering the cytokine storm is one of the essential strategies to save severely ill patients with ARDS from SARS-CoV-2 infection, and corticosteroid is a candidate drug to control cytokine storm syndrome after SARS-CoV-2 infection. However, there is insufficient evidence to recommend corticosteroid treatment according to the current interim guidance from WHO [17]. They 
concluded that administration of corticosteroid for acute severe lung injury from SARS-CoV and MARSCoV inhibits immune response and virus clearance, making the patient vulnerable to side effects such as psychosis, viremia, diabetes, avascular necrosis and secondary bacterial or fungal infection. Meanwhile, Shang et al. offered a negative perspective on use of corticosteroids for lung injury caused by SARS-CoV2 because the evidence supporting the effectiveness of corticosteroids came mostly from observational studies and was insufficient to conclude its usefulness [18]. Chen et al. supportively reported that proper use of corticosteroids reduced mortality and shortened the length of the hospital stay in critically ill patients without secondary infection and other complications [19]. Wu et al. reported that administration of methylprednisolone to patients with severe COVID-19 was effective and reduced the risk of death from ARDS [20]. In reference to the standard therapy of methylprednisolone for interstitial pneumonia [21], we used a new protocol of methylprednisolone therapy as described above. With the use of this protocol, all patients under the age of 70 survived from ARDS. Unfortunately, our protocol had a limited effect on elderly patients. More than half of the patients over age 70 died from complications due to long-term use of methylprednisolone including brain infarction, upper GI bleeding and secondary bacterial and fungal infections. Although the administration of methylprednisolone for severe pneumonia caused by SARSCoV-2 is controversial, our protocol exhibited a certain effect for limited cases.

Tocilizumab, an anti-IL-6 receptor antibody, has been approved for the cytokine release syndrome associated with CAR-T (chimeric antigen receptor-modified T cell) therapy [22]. Tocilizumab, along with steroids, is expected to control cytokine storm syndrome in COVID-19 [23]. In our case series, tocilizumab was administered to two patients prior to intubation, but it failed to improve oxygenation, and these patients required additional methylprednisolone administration. Thus, it was difficult to assess the effect of tocilizumab in the present study.

In intubated patients with severe ARDS, early and prolonged proning improves oxygenation and decreases mortality $[24,25]$. In adult patients with severe ARDS, prone ventilation for 12-16 hours per day is recommended also in COVID-19 [26]. Among the treatment methods introduced for the management of ARDS patients, proning can be used as an adjuvant therapy for improving respiratory function in these patients. Proning should not be a desperate final attempt but should be considered in the early stages of respiratory therapy [27] as the available evidence suggests that the early application of prolonged ventilation in the prone position decreases 28 - and 90-day mortality in patients with severe ARDS [17]. In our case series, 19 of the 20 patients were placed in a completely prone position with mechanical ventilation for 16 consecutive hours every day, which continued until improvement of respiratory condition or a life-threatening reason caused its cessation. The time from intubation to the first proning procedure was a median 1.8 (range 1-9) days, and the median number of procedures per person was 8.9 (range 0-24). Patients ventilated in the prone position face risks such as accidental removal of the tracheal tube, bending or pulling of catheters, pressure sores, facial edema, gastroesophageal reflux, and hypersalivation [28]. Several of our patients developed facial pressure sores, but no life-threatening complications occurred. We did not initiate ECMO in any of our patients because oxygenation was improved by proning. 


\section{Conclusion}

The present study showed the prognosis of COVID-19 patients who required invasive mechanical ventilation in a single Japanese intensive care center. Although the number of cases is small, the combination of antiviral and anti-inflammatory therapies and respiratory physiotherapy according to a single protocol were effective and resulted in a mortality rate of $30 \%$. It will be necessary to analyze more cases and develop standard treatments that improve the prognosis of severely ill patients with COVID-19.

\section{Abbreviations}

ACE2: Angiotensin converting enzyme 2; APACHE II: Acute Physiology and Chronic Health Evaluation II; ARDS: Acute respiratory distress syndrome; COVID-19: Coronavirus disease 2019; CT: Computed tomography; ECMO: Extracorporeal membrane oxygenation; IQR: interquartile range; KL: Krebs von den Lungen; RT-PCR: Reverse transcriptase polymerase chain reaction; SARS-CoV-2: Severe acute respiratory syndrome coronavirus 2; SOFA: Sequential Organ Failure Assessment; WHO: World Health Organization

\section{Declarations}

\section{Acknowledgements}

Not applicable.

\section{Authors' contributions}

HS and KY conceived, designed, and coordinated the study. HS wrote the first draft of this manuscript. KT, $\mathrm{YO}, \mathrm{HN}, \mathrm{HO}, \mathrm{YM}, \mathrm{SM}, \mathrm{SM}, \mathrm{SK}$, and HI contributed to the collection, analysis, and interpretation of the data and assisted in the preparation of the manuscript. DW, TY, FS and YN helped to draft the manuscript and revised it critically for important intellectual content. YK contributed to the final approval of the version to be published. All authors read and approved the final manuscript.

\section{Funding}

There are no sources of funding for this case report.

\section{Availability of data and materials}

This case report only contains clinical data from the medical records of the patient reported herein. The data will be made available upon request.

\section{Ethics approval and consent to participate}

Not applicable.

\section{Consent for publication}


Written informed consent was waived by the Ethics Committee due to the retrospective nature of this study and emergency circumstances of this infectious disease

\section{Competing interests}

The authors declare that they have competing interests.

\section{References}

1. World Health Organization. Novel coronavirus - China. http://www.who.int/csr/don/12-january -2020-novel-coronavirus-china/en/. Accessed 16 Jun 2020.

2. World Health Organization. WHO Coronavirus Disease (COVID-19) Dashboard. https://covid19.who.int/. Accessed 16 Jun 2020.

3. Ministry of Health. Labour and Welfare. https://www.mhlw.go.jp/stf/newpage_11621.html. Accessed 16 Jun 2020.

4. Zou X, Chen K, Zou J, Han P, Hao J, Han Z. Single-cell RNA-seq data analysis on the receptor ACE2 expression reveals the potential risk of different human organs vulnerable to 2019-nCoV infection. Front Med. 2020;14:185-92.

5. Mehta P, McAuley DF, Brown M, Sanchez E, Tattersall RS, Manson JJ, et al. COVID-19: consider cytokine storm syndromes and immunosuppression. Lancet. 2020;395:1033-4.

6. Huang C, Wang Y, Li X, Ren L, Zhao J, Hu Y, et al. Clinical features of patients infected with 2019 novel coronavirus in Wuhan, China. Lancet. 2020;395:497-506.

7. Yang X, Yu Y, Xu J, Shu H, Xia J, Liu H, et al. Clinical course and outcomes of critically ill patients with SARS-CoV-2 pneumonia in Wuhan, China: a single-centered, retrospective, observational study. Lancet Respir Med. 2020;8:475-81.

8. Grasselli G, Zangrillo A, Zanella A, Antonelli M, Cabrini L, Castelli A, et al. Baseline characteristics and outcomes of 1591 patients infected with SARS-CoV-2 admitted to ICUs of the Lombardy Region. Italy JAMA. 2020;323:1574-81.

9. Arentz M, Yim E, Klaff L, Lokhandwala S, Riedo FX, Chong M, et al. Characteristics and outcomes of 21 critically ill patients with COVID-19 in Washington State. JAMA. 2020;323:1612-4.

10. 1

0

10.1503/cmaj.200794

Mitra AR, Fergusson NA, Lloyd-Smith E, Wormsbecker A, Foster D, Karpov A, et al. Baseline characteristics and outcomes of patients with COVID-19 admitted to intensive care units in Vancouver, Canada: a case series. CMAJ 2020: [serial online]. https://doi.org/10.1503/cmaj.200794. Accessed 20 Jun 2020.

11. Ruan Q, Yang K, Wang W, Jiang L, Song J. Clinical predictors of mortality due to COVID-19 based on an analysis of data of 150 patients from Wuhan, China. Intensive Care Med. 2020;46:846-8. 
12. Richardson S, Hirsch JS, Narasimhan M, Crawford JM, McGinn T, Davidson KW, et al. Presenting characteristics, comorbidities, and outcomes among 5700 patients hospitalized with COVID-19 in the New York City Area. JAMA 2020;323:2052-9.

13. Wang M, Cao R, Zhang L, Yang X, Liu J, Xu M, et al. Remdesivir and chloroquine effectively inhibit the recently emerged novel coronavirus (2019-nCoV) in vitro. Cell Res 2020;30:269-71.

14. 1

4

10.1016/j.eng.2020.03.007

Cai Q, Yang M, Liu D, Chen J, Shu D, Xia J, et al. experimental treatment with favipiravir for COVID-19: an open-label control study. Engineering (Beijing) 2020: [serial online]. https://doi.org/10.1016/j.eng.2020.03.007. Accessed 20 Jun 2020.

15. Barnes GD, Burnett A, Allen A, Blumenstein M, Clark NP, Cuker A, et al. Thromboembolism and anticoagulant therapy during the COVID-19 pandemic: interim clinical guidance from the anticoagulation forum. J Thromb Thrombolysis. 2020;50:72-81.

16. Iwabuchi K, Yoshie K, Kurakami Y, Takahashi K, Kato Y, Morishima T. Therapeutic potential of ciclesonide inhalation for COVID-19 pneumonia: report of three cases. $J$ Infect Chemother. 2020;26:625-32.

17. Russell CD, Millar JE, Baillie JK. Clinical evidence does not support corticosteroid treatment for 2019nCoV lung injury. Lancet. 2020;395:473-5.

18. Shang L, Zhao J, Hu Y, Du R, Cao B. On the use of corticosteroids for 2019-nCoV pneumonia. Lancet. 2020;395:683-4.

19. Chen RC, Tang XP, Tan SY, Liang BL, Wan ZY, Fang JQ, et al. Treatment of severe acute respiratory syndrome with glucosteroids: the Guangzhou experience. Chest. 2006;129:1441-52.

20. Wu C, Chen X, Cai Y, Xia J, Zhou X, Xu S, et al. Risk factors associated with acute respiratory distress syndrome and death in patients with coronavirus disease 2019 pneumonia in Wuhan, China. JAMA Intern Med 2020: [serial online]. https://jamanetwork.com/journals/jamainternalmedicine/fullarticle/2763184. Accessed 20 Jun 2020.

21. Arai T, Tachibana K, Sugimoto C, Inoue Y, Tokura S, Okuma T, et al. High-dose prednisolone after intravenous methylprednisolone improves prognosis of acute exacerbation in idiopathic interstitial pneumonias. Respirology. 2017;22:1363-70.

22. Maude SL, Barrett D, Teachey DT, Grupp SA. Managing cytokine release syndrome associated with novel T cell-engaging therapies. Cancer J. 2014;20:119-22.

23. Xu X, Han M, Li T, Sun W, Wang D, Fu B, et al. Effective treatment of severe COVID-19 patients with tocilizumab. Proc Natl Acad Sci U S A. 2020;117:10970-5.

24. Munshi L, Del Sorbo L, Adhikari NKJ, Hodgson CL, Wunsch H, Meade MO, et al. Prone position for acute respiratory distress syndrome. a systematic review and meta-analysis. Ann Am Thorac Soc. 2017;14:280-8. 
25. Guérin C, Reignier J, Richard JC, Beuret P, Gacouin A, Boulain T, et al. Prone positioning in severe acute respiratory distress syndrome. N Engl J Med. 2013;368:2159-68.

26. World Health Organization. Clinical management of COVID-19.

https://www.who.int/publications/i/item/clinical-management-of-covid-19. Accessed 20 Jun 2020.

27. Xie H, Zhou ZG, Jin W, Yuan CB, Du J, Lu J, et al. Ventilator management for acute respiratory distress syndrome associated with avian influenza A (H7N9) virus infection: A case series. World J Emerg Med. 2018;9:118-24.

28. McCormick J, Blackwood B. Nursing the ARDS patient in the prone position: the experience of qualified ICU nurses. Intensive Crit Care Nurs. 2001;17:331-40.

\section{Tables}

Due to technical limitations the Tables are available as a download in the Supplementary Files.

\section{Figures}

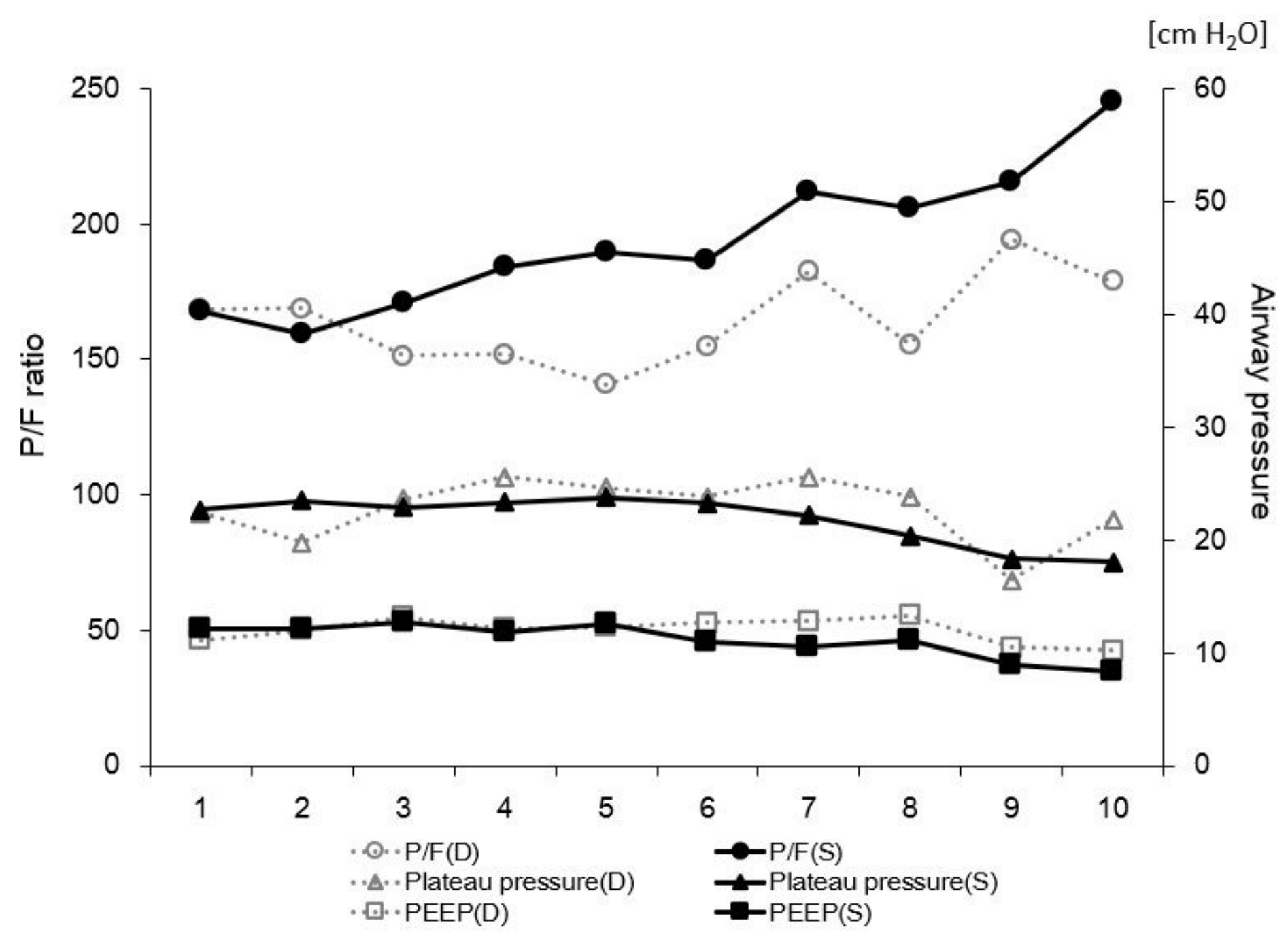


Figure 1

Daily trends of PaO2/FIO2 (P/F) ratio (circle), plateau pressure (triangle), and positive end-expiratory pressure (PEEP, square) during mechanical ventilation are indicated as sequential line graphs. Solid lines indicated data from the survival (S) group, and dotted lines indicate data from the non-survivor (D) group. The P/F ratio is scaled on the left vertical axis, and plateau pressure and PEEP are scaled on the right vertical axis
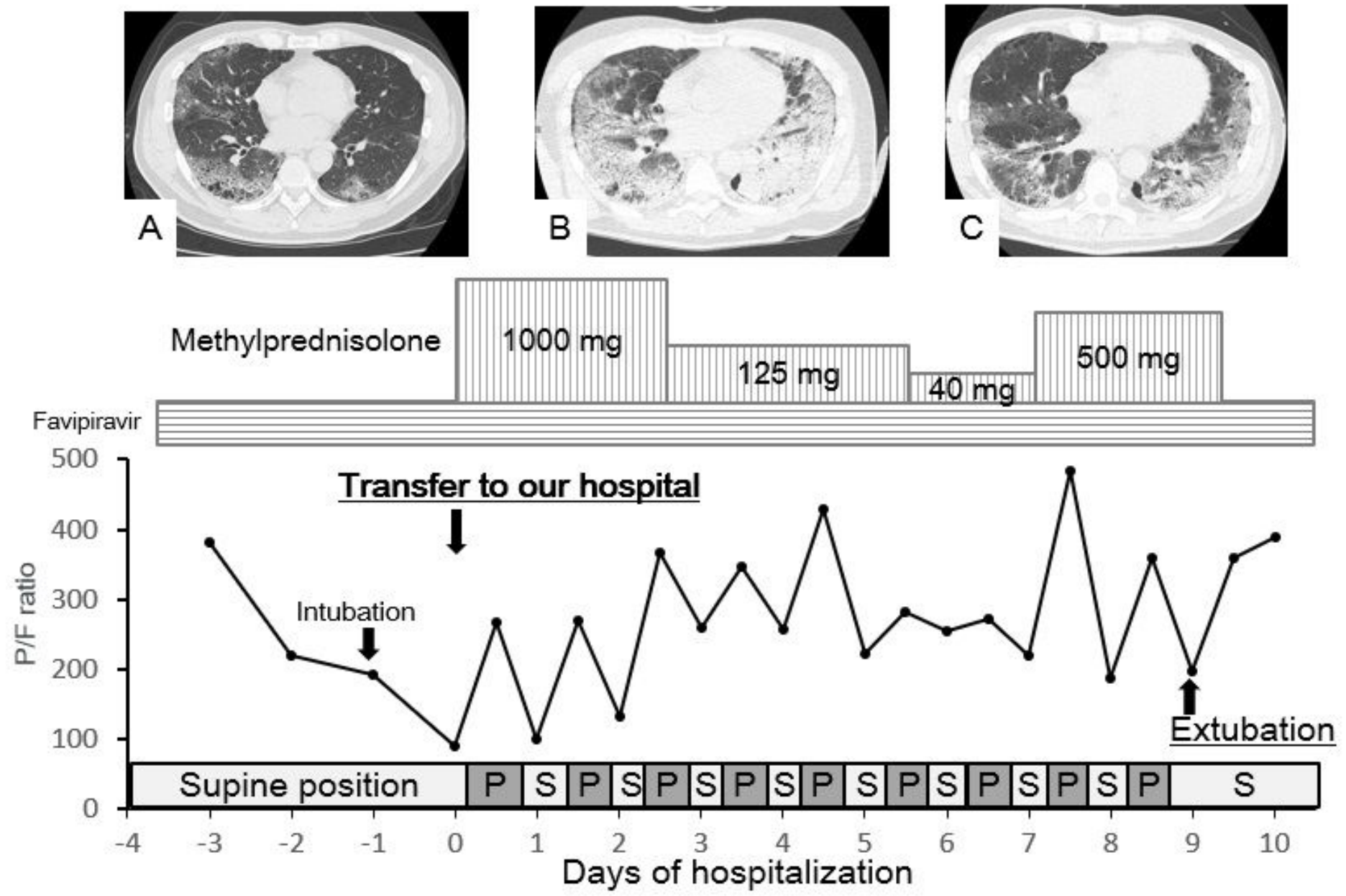

Figure 2

Treatment course in a 56 year-old man with pulmonary emphysema. His chief complaints were high fever, rhinorrhea, and fatigue. Polymerase chain reaction testing at a public health department was positive for COVID-19. He was treated for pneumonia caused by COVID-19 in a local hospital for 4 days.

Administration of favipiravir did not stop the deterioration of his respiratory insufficiency, and he was subsequently intubated and placed on mechanical ventilation. He was then transferred to our hospital for treatment of severe acute respiratory distress syndrome. On admission, his $\mathrm{P} / \mathrm{F}$ ratio was 89.5 , and thoracic $\mathrm{CT}$ revealed a diffuse ground-glass pattern with multiple bulla and blebs (A). Methylprednisolone pulse therapy and prone positioning began on day 1 in addition to administration of favipiravir and antibiotics. During the prone position, his P/F ratio tended to improve. On day 5 , his P/F ratio transiently 
worsened with expansion of ground-glass consolidations on CT (B). After additional half-pulse therapy with methylprednisolone for 3 days, his P/F ratio rose to $>400$. Finally, he could be extubated on day 10 , and the diffuse ground-glass pattern on thoracic CT began to fade away (C). P prone, S supine

\section{Supplementary Files}

This is a list of supplementary files associated with this preprint. Click to download.

- Tablesubmit.pptx 